\title{
A new approach for testing fuzzy hypotheses based on fuzzy data
}

\author{
Mohsen Arefi $^{1}$, S. Mahmoud Taheri ${ }^{2}$ \\ ${ }^{1}$ Department of Statistics, Faculty of Mathematical Sciences and Statistics, \\ University of Birjand, Birjand, Iran \\ E-mail: Arefi@Birjand.ac.ir \\ ${ }^{2}$ Department of Basic Engineering Science, College of Engineering, University of Tehran, \\ Tehran, P.O. Box 11365-4563, Iran, and \\ Department of Mathematical Sciences, Isfahan University of Technology, \\ Isfahan 84156-83111, Iran, and \\ E-mail:Taheri@cc.iut.ac.ir \\ Received 5 August 2012 \\ Accepted 27 October 2012
}

\begin{abstract}
A new approach for testing fuzzy hypotheses based on fuzzy data is introduced. According to the proposed approach, a method is first developed based on the defined fuzzy point estimation, which are then used to make a procedure for testing fuzzy hypotheses. This approach has been used to test simple, one-sided and two-sided fuzzy hypotheses. Two new criteria, called degree of acceptance (DA) and degree of rejection (DR), have been proposed to evaluate the test result. The application of the proposed method to lifetime testing is studied.
\end{abstract}

Keywords: Degree of acceptance (DA), Degree of rejection (DR), Fuzzy confidence interval, Fuzzy hypothesis, Testing hypothesis, Computing with words.

\section{Introduction}

In classical inferential statistics, testing hypothesis is one of the most important and most investigated problems. The common approaches depend on precise data, exact hypotheses, and exact parameters. However, in practical situations, different kinds of uncertainty are present, especially in situations with imprecise data and/or imprecise hypotheses.

For example, in reliability analysis, measuring the lifetime of a battery may not yield an exact result. A battery may work perfectly over a certain period but be losing in power for some time, and finally go dead completely at a certain time. In this case, we may re- port the lifetime with words rather than numbers, such as: about $1000(\mathrm{~h})$, approximately $1400 \mathrm{(h)}$, and so on. In addition, in the study of battery lifetime, we may like to test some non-precise (fuzzy) hypotheses. For instance, we may be interested in testing if the mean of lifetimes is "near to 1500 (h)" or, alternatively, it is much less than 1500 (h). As another area, in clinical treatments, some kind of diseases may be diagnosed by ill-defined criteria. For instance, the disease severity may be described as low, medium, high, very high, and so on. Similarly, in such studies, we may be interested in testing non-exact hypotheses. For example, we may like to test if the proportion of people infected

*Corresponding author, Tel.: (+98) 561 2502301; Fax: (+98) 5612502041. 
by a certain virus is high (e.g., about 0.80 ) or, alternatively, if much less than 0.80 . Many examples can be given from other fields in which the observed data and/or the hypotheses for testing are described in nonexact terms. New procedures will, therefore, be needed for the statistical analysis in such non-exact environments, based on soft computing methods ${ }^{1,2,3}$ and/or using the methods of computing with words ${ }^{4,5}$. In recent decades, much attention has been devoted to the development of statistical methods in non-exact environments, using the fuzzy set theory. In the following, a brief review will be presented of some works on hypothesis testing in fuzzy environments.

The problem of testing hypotheses with fuzzy data, first, was considered by Casals and Gil ${ }^{6}$, Casals et al. ${ }^{7}$, Grzegorzewski ${ }^{8}$, and $\mathrm{Wu}^{9}$. Körner ${ }^{10}$ proposed an asymptotic test for expectation of random fuzzy variables. Montenegro et al. ${ }^{11}$ studied the problem of two sample hypothesis tests of means of a fuzzy random variable. Arnold ${ }^{12,13}$, Taheri and Arefi ${ }^{14}$, and Taheri and Behboodian ${ }^{15,16}$ investigated the topic of testing statistical hypothesis when the hypotheses are presented by words rather than numbers. Arefi and Taheri ${ }^{17}$, Grzegorzewski ${ }^{18}$, Taheri and Behboodian ${ }^{19}$, and Torabi et al. ${ }^{20}$ studied the problem of testing statistical hypotheses when both hypotheses and observations are fuzzy (see also Kruse and Meyer ${ }^{21}$ ). A generalized version of Neyman-Pearson lemma for testing fuzzy hypotheses using r-levels is investigated by Torabi ${ }^{22}$. The p-value approach to testing hypothesis in fuzzy environments has been studied by Filzmoser and Viertl ${ }^{23}$ and Parchami et al. ${ }^{24,25}$. In addition, some hierarchical soft methods were developed for testing statistical hypotheses in fuzzy environments by Arefi and Taheri 26, Buckley ${ }^{27}$, Chachi et al. ${ }^{28}$, Grzegorzewski ${ }^{29}$, Grzegorzewski and Hryniewicz ${ }^{30}$, Hryniewicz ${ }^{31}$, and Taheri and Hesamian ${ }^{32,33}$. For more studies about statistical methods in fuzzy environments, see, e.g. Taheri 34 and Viertl ${ }^{35,36}$.

This article is organized as follows. After reviewing some necessary concepts and notations in the present section, we will define, in Section 2, the concepts of fuzzy point estimation and fuzzy confidence interval when the available observations are fuzzy rather than crisp. In Section 3, we will investigate an approach to testing non-fuzzy hypotheses with fuzzy observations based on Kahraman's work (see Kahraman et al. ${ }^{37}$ ). In Section 4, after introducing simple, one-sided and twosided fuzzy hypotheses, we will extend Kahraman et al.'s approach to the case when both the data available and the hypotheses of interest are fuzzy. A brief conclusion will be provided in Section 5 .

First, let us recall some preliminary concepts and necessary notations about fuzzy sets and fuzzy numbers. For more details, see, e.g. Kruse and Meyer ${ }^{21}$ and Klir and Yuan ${ }^{38}$.

We place a "tilde" over a symbol to denote a fuzzy set. So, $\widetilde{A}: X \rightarrow[0,1]$ represents the membership function of the fuzzy set $\widetilde{A}$. An $\alpha$-cut of $\widetilde{A}$, written $\widetilde{A}_{\alpha}$, is defined as $\widetilde{A}_{\alpha}=\{x \mid \widetilde{A}(x) \geqslant \alpha\}$, for $0<\alpha \leqslant 1$.

A fuzzy number $\widetilde{M}$ is a fuzzy subset of the real numbers satisfying:

i) $\widetilde{M}(x)=1$ for some $x$,

ii) $\widetilde{M}_{\alpha}$ is a closed, bounded interval for $0<\alpha \leqslant 1$.

The set of all fuzzy numbers will be denoted by $F N(R)$. A trapezoidal fuzzy number $(T F N) \widetilde{T}$, denoted by $\widetilde{T}=\left(a_{1}, a_{2}, a_{3}, a_{4}\right)_{T}$, is defined by four numbers $a_{1} \leqslant$ $a_{2} \leqslant a_{3} \leqslant a_{4}$ with the following membership function (see Fig. 1)

$$
\widetilde{T}(x)= \begin{cases}\frac{x-a_{1}}{a_{2}-a_{1}} & a_{1}<x \leqslant a_{2} \\ 1 & a_{2}<x \leqslant a_{3} \\ \frac{a_{4}-x}{a_{4}-a_{3}} & a_{3}<x \leqslant a_{4} \\ 0 & \text { otherwise }\end{cases}
$$

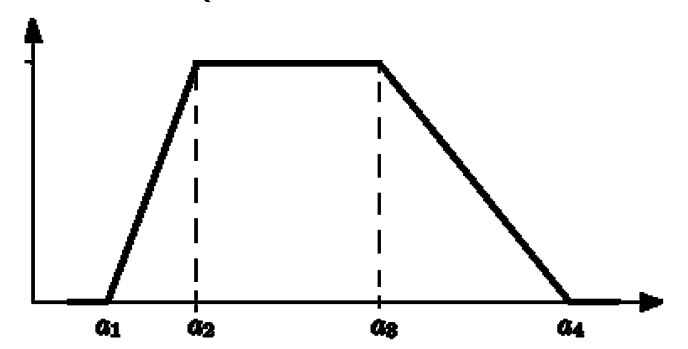

Fig. 1. Trapezoidal fuzzy number $\widetilde{T}=\left(a_{1}, a_{2}, a_{3}, a_{4}\right)_{T}$.

\section{Fuzzy confidence intervals}

Let $X_{1}, \ldots, X_{n}$ be a random sample of size $n$ from a probability density function (or probability mass function) $f(x ; \theta)$, with the observed values $x_{1}, \ldots, x_{n}$, where $\theta$ is the unknown parameter. Below, we investigate an 
approach to construct a fuzzy confidence interval for the unknown parameter $\theta$, when the available data are fuzzy. In this regard, we recall two well-known definitions from classical statistics (Definitions 1 and 3), to consider them as basis for extension to the fuzzy environment.

Definition 1 (see, Casella and Berger ${ }^{39}$ ) A function $Y=u\left(X_{1}, \ldots, X_{n}\right)$ which is independent of $\theta$ is called a point estimator for $\theta$. The observed value $\theta^{*}=$ $u\left(x_{1}, \ldots, x_{n}\right)$ is called the point estimation of $\theta$.

Definition 2 Suppose that, instead of $x_{1}, \ldots, x_{n}$, we can only get $\widetilde{X}_{1}, \ldots, \widetilde{X}_{n}$ as fuzzy observations. Then, by replacing the $\alpha$-cuts of $\widetilde{X}_{i}\left(\widetilde{X}_{i \alpha}\right)$ instead of $x_{i}$ in the point estimation $\theta^{*}$, and using the interval arithmetic, we obtain the $\alpha$-cuts of the fuzzy point estimation $\widetilde{\theta}^{*}$ as $\widetilde{\theta}_{\alpha}^{*}=u\left(\widetilde{X}_{1 \alpha}, \ldots, \widetilde{X}_{n \alpha}\right)$.

Remark 1 In this paper, for simplicity we consider $\widetilde{X}_{1}, \ldots, \widetilde{X}_{n}$ to be trapezoidal fuzzy numbers. Hence, $\widetilde{\theta}^{*}$ is a fuzzy number with the $\alpha$-cuts $\widetilde{\theta}_{\alpha}^{*}=\left[\widetilde{\theta}_{\alpha}^{* L}, \widetilde{\theta}_{\alpha}^{* U}\right]=$ $u\left(\widetilde{X}_{1 \alpha}, \ldots, \widetilde{X}_{n \alpha}\right)$.

Definition 3 (see, Casella and Berger ${ }^{39}$ ) Two-sided crisp confidence interval: Any interval $\left[\pi_{1}, \pi_{2}\right]$, where $\pi_{1}=\pi_{1}\left(\theta^{*}, \delta\right)$ and $\pi_{2}=\pi_{2}\left(\theta^{*}, \delta\right)$, which does not depend on $\theta$ and $P\left(\pi_{1} \leqslant \theta \leqslant \pi_{2}\right)=1-\delta$ is called a two-sided crisp confidence interval for $\theta$ at the confidence level of $1-\delta$.

Definition 4 (See Grzegorzewski ${ }^{8}$ and Kahraman et al. $\left.{ }^{37}\right)$ Two-sided fuzzy confidence interval: Consider a two-sided crisp confidence interval $\left[\pi_{1}, \pi_{2}\right]$ for $\theta$ at the confidence level of $1-\delta$. Suppose that we observe the fuzzy data $\widetilde{X}_{1}, \ldots, \widetilde{X}_{n}$. The $\alpha$-cuts $\Pi_{\alpha}=$ $\left[\Pi_{\alpha}^{L}, \Pi_{\alpha}^{U}\right]$ of the fuzzy confidence interval with the membership function $\mu_{\Pi}($.$) are defined as$

$\left\{\begin{array}{l}\Pi_{\alpha}^{L}=\min \left[\pi_{1}\left(\widetilde{\theta}_{\alpha}^{* L}, \delta\right), \pi_{1}\left(\widetilde{\theta}_{\alpha}^{* U}, \delta\right), \pi_{2}\left(\widetilde{\theta}_{\alpha}^{* L}, \delta\right), \pi_{2}\left(\widetilde{\theta}_{\alpha}^{* U}, \delta\right)\right], \\ \Pi_{\alpha}^{U}=\max \left[\pi_{1}\left(\widetilde{\theta}_{\alpha}^{* L}, \delta\right), \pi_{1}\left(\widetilde{\theta}_{\alpha}^{* U}, \delta\right), \pi_{2}\left(\widetilde{\theta}_{\alpha}^{* L}, \delta\right), \pi_{2}\left(\widetilde{\theta}_{\alpha}^{* U}, \delta\right)\right] .\end{array}\right.$

Definition 5 One-sided crisp confidence interval: Let $\underline{\pi}_{1}=\underline{\pi}_{1}\left(\theta^{*}, \delta\right)$ (or $\bar{\pi}_{2}=\bar{\pi}_{2}\left(\theta^{*}, \delta\right)$ ) be such that $P\left(\underline{\pi}_{1} \leqslant \theta\right)=1-\delta$ (or $\left.P\left(\theta \leqslant \bar{\pi}_{2}\right)=1-\delta\right)$. Then $\left[\underline{\pi}_{1}, \infty\right]\left(\right.$ or $\left.\left[-\infty, \bar{\pi}_{2}\right]\right)$ is called a lower (or upper) onesided confidence interval for $\theta$ at a confidence level of $(1-\delta)$.

Definition 6 (See Grzegorzewski ${ }^{8}$ and Kahraman et al. $\left.{ }^{37}\right)$ One-sided fuzzy confidence interval: Sup- pose that we have the one-sided crisp confidence intervals $\left[\underline{\pi}_{1}, \infty\right]$ and $\left[-\infty, \bar{\pi}_{2}\right]$. The $\alpha$-cuts $\Pi_{\alpha}$ of the lower and upper one-sided fuzzy confidence intervals are defined as follows

$$
\Pi_{\alpha}=\left[\underline{\Pi}_{\alpha}^{L}, \infty\right]=\left[\min \left[\underline{\pi}_{1}\left(\widetilde{\theta}_{\alpha}^{* L}, \delta\right), \underline{\pi}_{1}\left(\widetilde{\theta}_{\alpha}^{* U}, \delta\right)\right], \infty\right],
$$

and

$$
\Pi_{\alpha}=\left[-\infty, \bar{\Pi}_{\alpha}^{U}\right]=\left[-\infty, \max \left[\bar{\pi}_{2}\left(\widetilde{\theta}_{\alpha}^{* L}, \delta\right), \bar{\pi}_{2}\left(\widetilde{\theta}_{\alpha}^{* U}, \delta\right)\right]\right] .
$$

Note: For the numerical examples in this paper, we take the confidence level of $(1-\delta)$ equal to 0.95 .

Remark 2 Viertl ${ }^{35}$ proposed a method to define fuzzy confidence regions based on fuzzy data. His method is more general than ours in the sense that he does not assume the fuzzy data to be necessarily fuzzy numbers. In his approach, the data can be any normalized fuzzy subsets of the real line. Inside, Chachi and Taheri ${ }^{40}$ investigated the problem of constructing fuzzy confidence interval for fuzzy parameter, based on the h-cuts of normal (Gaussian) fuzzy random sample. It is remarkable that, in their method, they, first, obtained two different cases of the h-cuts of fuzzy confidence interval for $h$-cuts of fuzzy parameter, and then they introduced a method based on these h-cuts to obtain the membership function of the fuzzy confidence interval. But, in our method, we first obtain a fuzzy point estimation, and then the $\alpha$-cuts of fuzzy confidence interval is calculated based on the h-cuts of the fuzzy point estimation.

Example 1 Suppose that we have taken a fuzzy random sample from $N\left(\theta, \sigma^{2}=1\right)$ and we observed the following trapezoidal fuzzy data:

\begin{tabular}{||c|c||c|c||}
\hline No. & Data & No. & Data \\
\hline 1 & $(65,70,70,80)_{T}$ & 6 & $(65,70,80,85)_{T}$ \\
2 & $(55,65,70,75)_{T}$ & 7 & $(50,60,70,82)_{T}$ \\
3 & $(60,70,75,83)_{T}$ & 8 & $(55,62,75,85)_{T}$ \\
4 & $(55,60,60,65)_{T}$ & 9 & $(58,65,65,75)_{T}$ \\
5 & $(50,63,65,72)_{T}$ & & \\
\hline
\end{tabular}

Then, the two-sided crisp confidence interval for $\theta$ at the confidence level of $(1-\delta)$ is

$$
\left[\pi_{1}, \pi_{2}\right]=\left[\bar{X}-z_{1-\delta / 2} \frac{1}{\sqrt{n}}, \bar{X}+z_{1-\delta / 2} \frac{1}{\sqrt{n}}\right] .
$$

Now, based on Definition 2, by substituting $\left(\widetilde{\bar{X}}_{\alpha}\right)^{L}$ and $\left(\widetilde{\bar{X}}_{\alpha}\right)^{U}$ in $\bar{X}$, the two-sided fuzzy confidence interval for 
$\theta$ at a confidence level of 0.95 can yield a fuzzy set with the following $\alpha$-cuts

$$
\begin{aligned}
\Pi_{\alpha}= & {\left[\Pi_{\alpha}^{L}, \Pi_{\alpha}^{U}\right] } \\
= & {\left[\operatorname { m i n } \left[\left(\tilde{\bar{X}}_{\alpha}\right)^{L}-z_{1-\delta / 2} \frac{1}{\sqrt{n}},\left(\tilde{\bar{X}}_{\alpha}\right)^{U}-z_{1-\delta / 2} \frac{1}{\sqrt{n}},\right.\right.} \\
& \left.\left(\tilde{\bar{X}}_{\alpha}\right)^{L}+z_{1-\delta / 2} \frac{1}{\sqrt{n}},\left(\tilde{\bar{X}}_{\alpha}\right)^{U}+z_{1-\delta / 2} \frac{1}{\sqrt{n}}\right] \\
& \max \left[\left(\tilde{\bar{X}}_{\alpha}\right)^{L}-z_{1-\delta / 2} \frac{1}{\sqrt{n}},\left(\tilde{\bar{X}}_{\alpha}\right)^{U}-z_{1-\delta / 2} \frac{1}{\sqrt{n}},\right. \\
& \left.\left.\left(\tilde{\bar{X}}_{\alpha}\right)^{L}+z_{1-\delta / 2} \frac{1}{\sqrt{n}},\left(\tilde{\bar{X}}_{\alpha}\right)^{U}+z_{1-\delta / 2} \frac{1}{\sqrt{n}}\right]\right] \\
= & {\left[\left(\tilde{\bar{X}}_{\alpha}\right)^{L}-z_{1-\delta / 2} \frac{1}{\sqrt{n}},\left(\tilde{\bar{X}}_{\alpha}\right)^{U}+z_{1-\delta / 2} \frac{1}{\sqrt{n}}\right] } \\
= & {[56.35+8 \alpha, 78.65-8 \alpha], }
\end{aligned}
$$

where $\widetilde{\bar{X}}=(57,65,70,78)_{T}$ and its $\alpha$-cut is $\widetilde{\bar{X}}_{\alpha}=$ $\left[\left(\widetilde{\bar{X}}_{\alpha}\right)^{L},\left(\widetilde{\bar{X}}_{\alpha}\right)^{U}\right]=[57+8 \alpha, 78-8 \alpha]$.

In this example, the lower and upper one-sided crisp confidence intervals for $\theta$ at a confidence level of $(1-\delta)$ are $\left[\underline{\pi}_{1}, \infty\right]=\left[\bar{X}-z_{1-\delta} \frac{1}{\sqrt{n}}, \infty\right]$ and $\left[-\infty, \bar{\pi}_{2}\right]=$ $\left[-\infty, \bar{X}+z_{1-\delta} \frac{1}{\sqrt{n}}\right]$.

By substituting $\left(\widetilde{\bar{X}}_{\alpha}\right)^{L}$ and $\left(\widetilde{\bar{X}}_{\alpha}\right)^{U}$ in the one-sided crisp confidence intervals, we obtain the lower and upper one-sided fuzzy confidence intervals for $\theta$ at a confidence level of 0.95 as the fuzzy sets. The $\alpha$-cuts of these fuzzy sets are

$$
\begin{aligned}
\Pi_{\alpha} & =\left[\underline{\Pi}_{\alpha}^{L}, \infty\right] \\
& =\left[\min \left[(\tilde{\bar{X}})_{\alpha}^{L}-z_{1-\delta / 2} \frac{1}{\sqrt{n}},(\tilde{\bar{X}})_{\alpha}^{U}-z_{1-\delta / 2} \frac{1}{\sqrt{n}}\right], \infty\right] \\
& =\left[(\tilde{\bar{X}})_{\alpha}^{L}-z_{1-\delta / 2} \frac{1}{\sqrt{n}}, \infty\right]=[56.45+8 \alpha, \infty], \quad(5)
\end{aligned}
$$

and

$$
\begin{aligned}
\Pi_{\alpha} & =\left[-\infty, \bar{\Pi}_{\alpha}^{U}\right] \\
& =\left[-\infty, \max \left[(\tilde{\bar{X}})_{\alpha}^{L}+z_{1-\delta} \frac{1}{\sqrt{n}},(\tilde{\bar{X}})_{\alpha}^{U}+z_{1-\delta} \frac{1}{\sqrt{n}}\right]\right] \\
& =\left[-\infty,(\widetilde{\bar{X}})_{\alpha}^{U}+z_{1-\delta} \frac{1}{\sqrt{n}}\right]=[-\infty, 78.55-8 \alpha] .(6)
\end{aligned}
$$

Remark 3 Note that, in a general setting, it is not necessary for $\widetilde{\boldsymbol{\theta}}^{*}$ to be a fuzzy number. If $\widetilde{\boldsymbol{\theta}}^{*}$ is a fuzzy set, then we can follow the proposed procedure to construct the fuzzy confidence region instead of the fuzzy confidence interval.

\section{Testing hypotheses based on fuzzy data}

In this section, based on fuzzy observations $\widetilde{X}_{1}, \ldots, \widetilde{X}_{n}$ from a probability density function (or probability mass function) $f(x ; \theta)$, we review an approach to test the following hypotheses ${ }^{8,37}$ (we will extend this approach to fuzzy hypotheses in next section):
a) $\left\{\begin{array}{l}H_{0}: \theta=\theta_{0} \\ H_{1}: \theta \neq \theta_{0}\end{array}\right.$
b) $\left\{\begin{array}{l}H_{0}: \theta \leqslant \theta_{0} \\ H_{1}: \theta>\theta_{0}\end{array}\right.$
c) $\left\{\begin{array}{l}H_{0}: \theta \geqslant \theta_{0} \\ H_{1}: \theta<\theta_{0}\end{array}\right.$

Definition 7 For testing the above hypotheses based on fuzzy data $\widetilde{X}_{1}, \ldots, \widetilde{X}_{n}$, a function $\Phi:(F N(R))^{n} \rightarrow$ $P(\{0,1\})$ given by

$\Phi\left(\widetilde{X}_{1}, \ldots, \widetilde{X}_{n}\right)= \begin{cases}\{0\} & \text { if } \theta_{0} \in\left(S_{\Pi} \cap\left(S_{-\Pi}\right)^{c}\right) \\ \{1\} & \text { if } \theta_{0} \in\left(S_{-\Pi} \cap\left(S_{\Pi}\right)^{c}\right) \\ \{0,1\} & \text { if } \theta_{0} \in\left(S_{-\Pi} \cap S_{\Pi}\right)\end{cases}$

is called a fuzzy test function at the significance level of $\delta$, where in the third part, $H_{0}$ is accepted with a degree of acceptance (DA) equal to $\mu_{\Pi}\left(\theta_{0}\right)$ and is rejected with a degree of rejection $(D R)$ equal to $\mu_{-\Pi}\left(\theta_{0}\right)$. For hypotheses $a, b$, and $c$, the membership function of $\Pi$ is obtained from relations (1), (2), and (3), respectively. Also, $P(\{0,1\})$ is the power set of $\{0,1\}$, and $S_{\Pi}$ and $S_{-\Pi}$ are the supports of the fuzzy sets $\mu_{\Pi}(x)$ and $\mu_{-\Pi}(x)=1-\mu_{\Pi}(x)$, respectively. (See Fig. 2-4 for the cases $a, b$, and $c$ ).

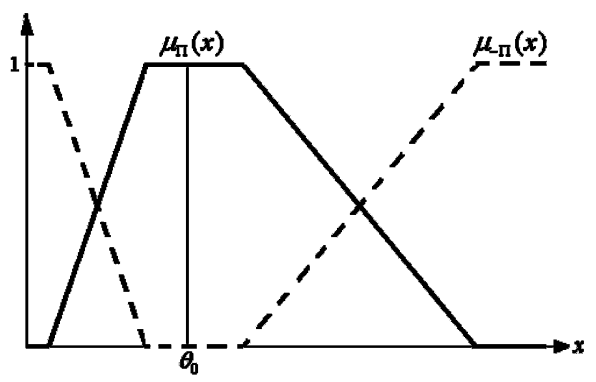

Fig. 2. $H_{0}$ is accepted with $\mathrm{DA}=1$. 


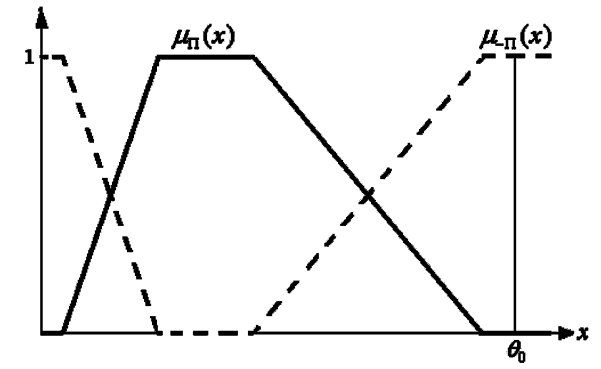

Fig. 3. $H_{0}$ is rejected with $\mathrm{DR}=1$.

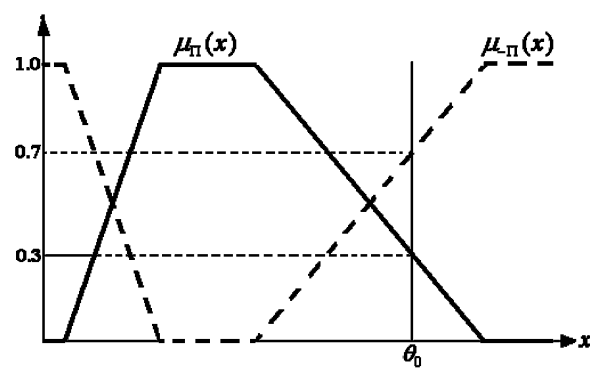

Fig. 4. $H_{0}$ is accepted (rejected) with $\mathrm{DA}=0.3(\mathrm{DR}=0.7)$.

Remark 4 The above procedure can be reduced to the ordinary procedure for testing hypotheses, if the data set is crisp (non-fuzzy).

Example 2 Consider Example 1. Suppose that we are interested in testing the following hypotheses at a significance level of $\delta=0.05$

$$
\left\{\begin{array}{l}
H_{0}: \theta=75 \\
H_{1}: \theta \neq 75
\end{array}\right.
$$

Based on Relation (4), the membership function $\mu_{\Pi}(x)$ is obtained as

$$
\mu_{\Pi}(x)= \begin{cases}\frac{3 x-169.04}{24} & 56.35 \leqslant x<64.35 \\ 1 & 64.35 \leqslant x<70.65 \\ \frac{235.96-3 x}{24} & 70.65 \leqslant x<78.65\end{cases}
$$

Hence, $H_{0}$ is accepted with $D A=0.46$ and is rejected with $D R=0.54$ (Fig. 5).

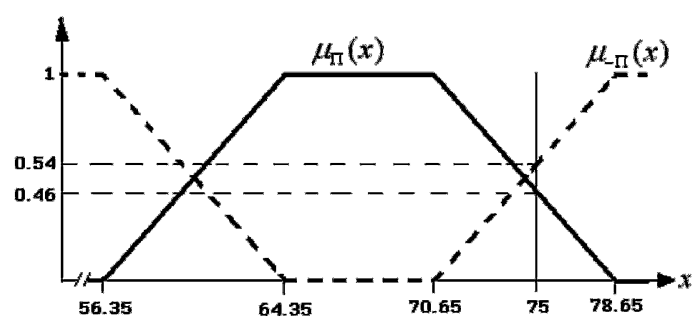

Fig. 5. $H_{0}$ is rejected (accepted) with $\mathrm{DR}=0.54(\mathrm{DA}=0.46)$.
Example 3 Consider Example 1. Suppose that we want to test the following one-sided hypotheses:

$$
\left\{\begin{array}{l}
H_{0}: \theta \leqslant 75 \\
H_{1}: \theta>75 .
\end{array}\right.
$$

Based on Relation (5), the membership function $\mu_{\Pi}(x)$ of the one-sided fuzzy confidence interval is obtained as

$$
\mu_{\Pi}(x)= \begin{cases}\frac{x-56.45}{8} & 56.45 \leqslant x<64.45 \\ 1 & 64.45 \leqslant x \\ 0 & \text { otherwise. }\end{cases}
$$

Hence, at the significance level of $\delta=0.05$, we accept $H_{0}$ with $D A=1$. (See Fig. 6).

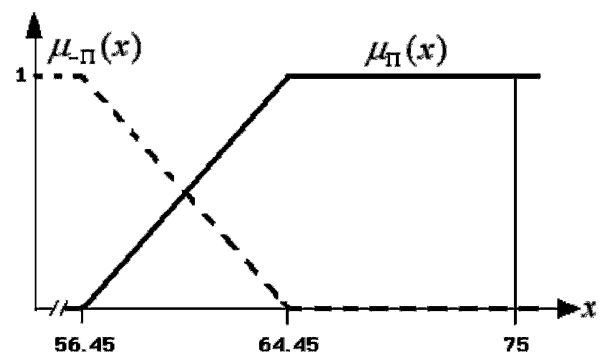

Fig. 6. $H_{0}$ is accepted with $\mathrm{DA}=1$.

Example 4 Consider Example 1. Suppose that we want to test the following one-sided hypotheses:

$$
\left\{\begin{array}{l}
H_{0}: \theta \geqslant 75 \\
H_{1}: \theta<75
\end{array}\right.
$$

Based on Relation (6), the membership function $\mu_{\Pi}(x)$ of the one-sided fuzzy confidence interval is obtained as

$$
\mu_{\Pi}(x)= \begin{cases}1 & x<70.55 \\ \frac{78.55-x}{8} & 70.55 \leqslant x<78.55 \\ 0 & \text { otherwise }\end{cases}
$$

Hence, at the significance level of $\delta=0.05$, we accept $H_{0}$ with $D A=0.44$. (See Fig. 7).

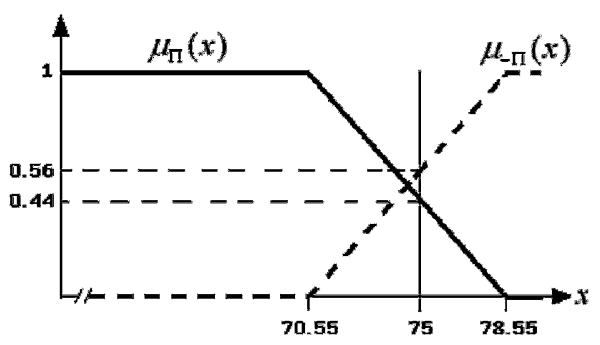

Fig. 7. $H_{0}$ is accepted with $\mathrm{DA}=0.44$. 


\section{Testing fuzzy hypotheses based on fuzzy data}

In this section, we extend the concepts of simple and composite hypotheses to simple and composite fuzzy hypotheses. Then, we investigate a new approach for testing hypotheses based on fuzzy data when the hypotheses of interest are fuzzy, too.

Definition 8 Let $\widetilde{\theta}_{0}$ be a known fuzzy number.

i) Any hypothesis of the form $\left(H: \theta\right.$ is $\left.\widetilde{\theta}_{0}\right)$ is called a fuzzy simple hypothesis.

ii) Any hypothesis of the form $\left(H: \theta\right.$ is not $\left.\widetilde{\theta}_{0}\right)$ is called a fuzzy two-sided hypothesis.

iii) Any hypothesis of the form ( $H$ : $\theta$ is smaller than $\left.\widetilde{\theta}_{0}\right)$ is called a fuzzy left one-sided hypothesis.

iv) Any hypothesis of the form ( $H$ : $\theta$ is larger than $\left.\widetilde{\theta}_{0}\right)$ is called a fuzzy right one-sided hypothesis.

In the following, we investigate some methods to test the hypotheses in the following forms
$\left.a^{\prime}\right)\left\{\begin{array}{l}H_{0}: \theta \text { is } \widetilde{\theta}_{0}, \\ H_{1}: \theta \text { is not } \widetilde{\theta}_{0},\end{array}\right.$
$\left.b^{\prime}\right)\left\{\begin{array}{l}H_{0}: \theta \text { is larger than } \widetilde{\theta}_{0}, \\ H_{1}: \theta \text { is not larger than } \widetilde{\theta}_{0},\end{array}\right.$
$\left.c^{\prime}\right)\left\{\begin{array}{l}H_{0}: \theta \text { is smaller than } \widetilde{\theta}_{0}, \\ H_{1}: \theta \text { is not smaller than } \widetilde{\theta}_{0} .\end{array}\right.$

For simplicity, we will consider the fuzzy number $\widetilde{\theta}_{0}$ to be a trapezoidal fuzzy number $\widetilde{\theta}_{0}=$ $\left(a_{1}, a_{2}, a_{3}, a_{4}\right)_{T}$. For constructing our method, we assume that

- $A_{T}$ is the total area under the graph of $\widetilde{\theta}_{0}$.

- $A_{\Pi}$ is the area under both the graph $\mu_{\Pi}(x)$ and the graph $\widetilde{\theta}_{0}$, limited to the interval $\left[\Pi_{0.5}^{L}, \Pi_{0.5}^{U}\right]$.
- $A_{-\Pi}$ is the area under both the graph $\mu_{-\Pi}(x)$ and the graph $\widetilde{\theta}_{0}$, limited to the interval $\left[-\infty, \Pi_{0.5}^{L}\right] \cup\left[\Pi_{0.5}^{U}, \infty\right]$.

The above areas are shown in Fig. 8-10 for the hypotheses $a^{\prime}, b^{\prime}$ and $c^{\prime}$, respectively.

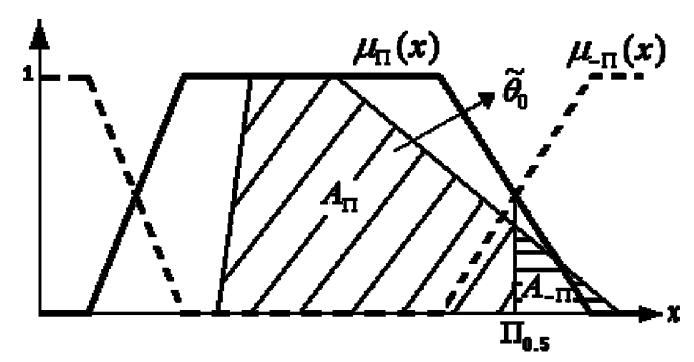

Fig. 8. $A_{\Pi}$ and $A_{-\Pi}$ in case $a^{\prime}$.

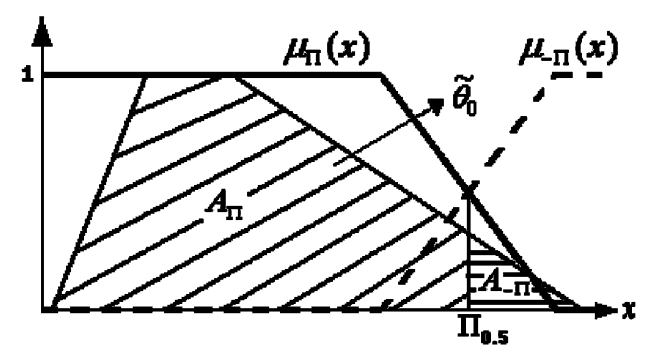

Fig. 9. $A_{\Pi}$ and $A_{-\Pi}$ in case $b^{\prime}$.

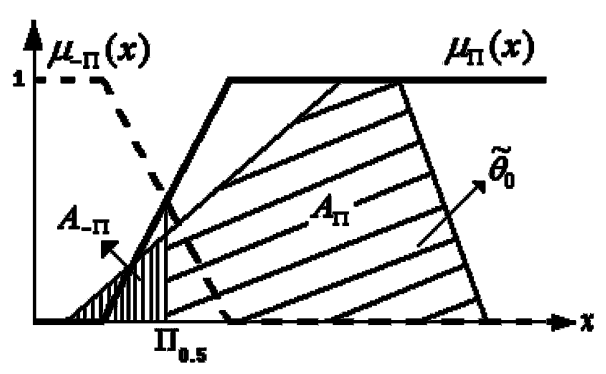

Fig. 10. $A_{\Pi}$ and $A_{-\Pi}$ in case $c^{\prime}$.

Definition 9 For the above one-sided and two-sided fuzzy hypotheses and based on fuzzy data $\widetilde{X}_{1}, \ldots, \widetilde{X}_{n}, a$ function $\Phi:(F N(R))^{n} \rightarrow P(\{0,1\})$ given by

$\Phi\left(\widetilde{X}_{1}, \widetilde{X}_{2}, \ldots, \widetilde{X}_{n}\right)= \begin{cases}\{0\} & \text { if }\left[a_{1}, a_{4}\right] \subseteq \Pi_{0.5}, \\ \{1\} & \text { if }\left[a_{1}, a_{4}\right] \subseteq\left(-\Pi_{0.5}\right), \\ \{0,1\} & \text { otherwise, }\end{cases}$

is called to be a fuzzy test function at the significance level of $\delta$. In the third part of Relation (11), $H_{0}$ is accepted with a degree of acceptance $D A=\frac{A_{\Pi}}{A_{T}}$ and is 
rejected with a degree of rejection $D R=\frac{A_{-\Pi}}{A_{T}}$. For hypotheses $a^{\prime}, b^{\prime}$ and $c^{\prime}, \Pi_{0.5}$ is obtained from Relations (1), (2) and (3), respectively. Also, $\left[a_{1}, a_{4}\right]$ is the support of the fuzzy number $\widetilde{\theta}_{0}$.

Remark 5 The motivation behind the above definition is the one-to-one correspondence between confidence sets and tests of hypotheses (see, e.g. Theorem 9.2.1 (Section 9) in Casella and Berger ${ }^{39}$ ). For example, in testing the hypothesis $H_{0}: \theta=\theta_{0}$ vs. $H_{1}: \theta \neq \theta_{0}$, we accept $H_{0}$ if $\theta_{0}$ belongs to the (same level) confidence interval and reject $H_{0}$ if not. Now, in the fuzzy case, we accept the fuzzy hypothesis " $H_{0}: \theta$ is $\widetilde{\theta}_{0}$ ” by a degree of acceptance (DA). This degree of acceptance, is calculated based on the area under the graph of $\widetilde{\theta}_{0}$ which is under the graph of $\mu_{\Pi}($.$) (the membership function$ of the fuzzy confidence interval).

Example 5 Consider Example 1. Now suppose we would like to test the following fuzzy hypotheses at the significance level of $\delta=0.05$

$$
\left\{\begin{array}{l}
H_{0}: \theta \text { is }(65,70,76,81)_{T}, \\
H_{1}: \theta \text { is not }(65,70,76,81)_{T} .
\end{array}\right.
$$

Here, $H_{0}$ suggests that $\theta$ is almost between 70 and 76 , and $H_{1}$ suggests that $\theta$ is away from the interval [70,76]. As in Example 2, the membership function $\mu_{\Pi}(x)$ is obtained in the following way:

$$
\mu_{\Pi}(x)= \begin{cases}\frac{3 x-169.04}{24} & \text { if } 56.35 \leqslant x<64.35 \\ 1 & \text { if } 64.35 \leqslant x<70.65 \\ \frac{235.96-3 x}{24} & \text { if } 70.65 \leqslant x<78.65 \\ 0 & \text { otherwise. }\end{cases}
$$

Hence, by Definition 9, at the significance level $\delta=$ 0.05, $H_{0}$ is accepted with $D A=\frac{A_{\Pi}}{A_{T}}=\frac{6.15}{11}=0.56$ and is rejected with $D R=\frac{A_{-\Pi}}{A_{T}}=\frac{3.12}{11}=0.28$. (See Fig. 11).

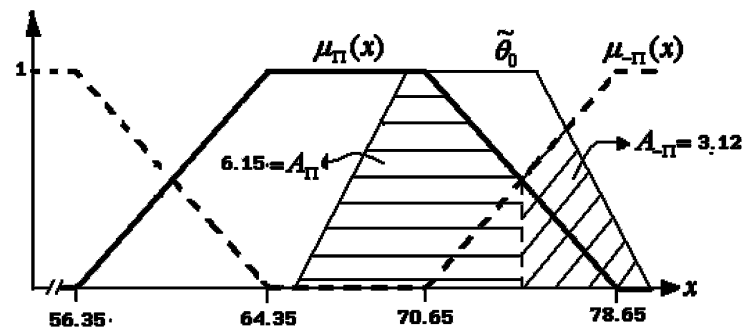

Fig. 11. $H_{0}$ is accepted (rejected) with $D A=0.56(D R=0.28)$.
Example 6 Consider Example 1. We are interested in testing the following one-sided fuzzy hypotheses at a significance level of $\delta=0.05$

$$
\left\{\begin{array}{l}
H_{0}: \theta \text { is larger than }(65,70,76,81)_{T}, \\
H_{1}: \theta \text { is not larger than }(65,70,76,81)_{T} .
\end{array}\right.
$$

In Example 3, the membership function $\mu_{\Pi}(x)$ is obtained as

$$
\mu_{\Pi}(x)= \begin{cases}1 & \text { if } x \leqslant 70.55 \\ \frac{78.55-x}{8} & \text { if } 70.55<x \leqslant 78.55 \\ 0 & \text { otherwise. }\end{cases}
$$

Therefore, by Definition 9, at the significance level of $\delta=0.05, H_{0}$ is accepted with $D A=\frac{A_{\Pi}}{A_{T}}=\frac{6.05}{11}=0.55$ and is rejected with $D R=\frac{A_{-\Pi}}{A_{T}}=\frac{3.20}{11}=0.29$. (See Fig. 12).

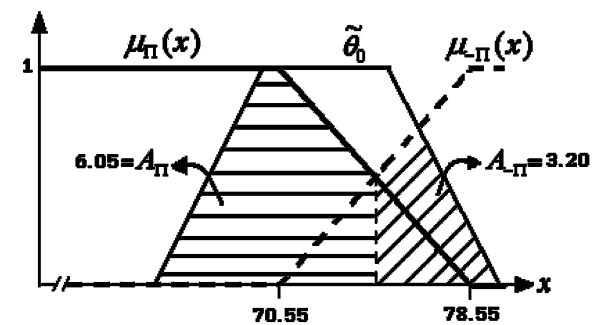

Fig. 12. $H_{0}$ is accepted (rejected) with $D A=0.55(D R=0.29)$.

Example 7 Consider Example 1. Suppose we want to test the following one-sided fuzzy hypotheses at the significance level of $\delta=0.05$

$$
\left\{\begin{array}{l}
H_{0}: \theta \text { is smaller than }(65,70,76,81)_{T}, \\
H_{1}: \theta \text { is not smaller than }(65,70,76,81)_{T} .
\end{array}\right.
$$

In Example 4, the membership function $\mu_{\Pi}(x)$ is obtained as

$$
\mu_{\Pi}(x)= \begin{cases}\frac{x-56.45}{8} & \text { if } 56.45 \leqslant x<64.45 \\ 1 & \text { if } 64.45 \leqslant x \\ 0 & \text { otherwise. }\end{cases}
$$

Therefore, by Definition 9, at a significance level of $\delta=0.05, H_{0}$ is accepted with $D A=\frac{A_{\Pi}}{A_{T}}=1$ and is rejected with $D R=\frac{A_{-\Pi}}{A_{T}}=0$. (See Fig. 13). 


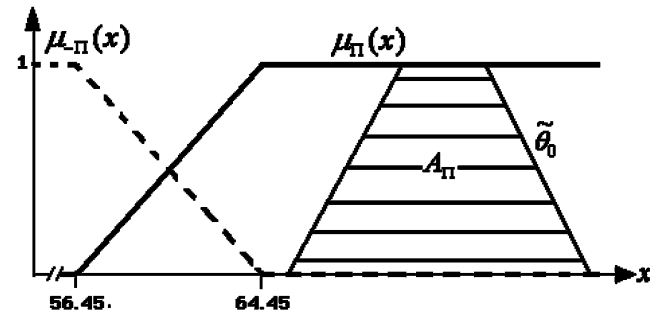

Fig. 13. $H_{0}$ is accepted (rejected) with $D A=1(D R=0)$.

Example 8 Application in lifetime testing: The lifetime of the batteries (in terms of hours) produced by a company has an exponential distribution with an unknown mean $\theta$, i.e.

$$
f(x ; \theta)=\frac{1}{\theta} \exp \left\{-\frac{x}{\theta}\right\}, \quad x>0, \quad \theta>0 .
$$

In a random sample of size $n=12$ batteries, we observed the following trapezoidal fuzzy data:

\begin{tabular}{||c|c||c|c||}
\hline No & Data & No & Data \\
\hline 1 & $(0,0,700,1200)_{T}$ & 7 & $(0,0,100,700)_{T}$ \\
2 & $(0,0,1050,1100)_{T}$ & 8 & $(0,0,900,1150)_{T}$ \\
3 & $(0,0,700,1050)_{T}$ & 9 & $(0,0,550,1100)_{T}$ \\
4 & $(0,0,100,460)_{T}$ & 10 & $(0,0,150,700)_{T}$ \\
5 & $(0,0,1050,1300)_{T}$ & 11 & $(0,0,1100,1220)_{T}$ \\
6 & $(0,0,700,1180)_{T}$ & 12 & $(0,0,100,600)_{T}$ \\
\hline
\end{tabular}

Note that measuring the lifetime of a battery may not yield an exact number. A battery (for instance data No. 1) may work perfectly for 700 (h) before it begins to lose power during the next $500(h)$. It finally goes completely dead after 1200 (h). Suppose we are interested in testing the following one-sided fuzzy hypothesis at the significance level of $\delta=0.10$ :

$\left\{H_{0}: \theta\right.$ is larger than $(800,1000,1000,1250)_{T}$, $\left\{H_{1}: \theta\right.$ is not larger than $(800,1000,1000,1250)_{T}$.

The right one-sided crisp confidence interval for $\theta$ at a confidence level of $(1-\delta)$ is

$$
\left(0, \bar{\pi}_{2}\right]=\left(0, \frac{2 n \bar{X}}{\chi_{(2 n, \delta)}^{2}}\right] .
$$

Now, by substituting $\left(\widetilde{\bar{X}}_{\alpha}\right)^{L}$ and $\left(\widetilde{\bar{X}}_{\alpha}\right)^{U}$ of $\widetilde{\bar{X}}$ instead of $\bar{X}$ in the above relation, the upper one-sided fuzzy confidence interval for $\theta$ at a confidence level of $1-\delta=$
0.90 , is a fuzzy set with the following $\alpha$-cuts

$$
\begin{aligned}
\Pi_{\alpha}=\left(0, \bar{\Pi}_{\alpha}^{U}\right] & =\left(0, \max \left[\frac{2 n\left(\tilde{\bar{X}}_{\alpha}\right)^{L}}{\chi_{(2 n, \delta)}^{2}}, \frac{2 n\left(\tilde{\bar{X}}_{\alpha}\right)^{U}}{\chi_{(2 n, \delta)}^{2}}\right]\right] \\
& =(0,1502.042-582.424 \alpha],
\end{aligned}
$$

where the membership function of $\widetilde{\bar{X}}$ is $\mu_{\tilde{\bar{X}}}(x) \widehat{=}(0,0,600,980)_{T}$ and its $\alpha$-cut is $\left[\left(\widetilde{\bar{X}}_{\alpha}\right)^{L},\left(\widetilde{\bar{X}}_{\alpha}\right)^{U}\right]=[0,980-380 \alpha]$.

Based on Relation (12), the membership function $\mu_{\Pi}(x)$ is obtained as

$\mu_{\Pi}(x)= \begin{cases}1 & \text { if } 0 \leqslant x<919.618 \\ \frac{1502.042-x}{582.424} & \text { if } 919.618 \leqslant x<1502.042 \\ 0 & \text { otherwise. }\end{cases}$

Therefore, by Definition 9, at the significance level of $\delta=0.1, H_{0}$ is accepted with $D A=\frac{A_{\Pi}}{A_{T}}=\frac{216.342}{225}=$ 0.962 and is rejected with $D R=\frac{A_{-\Pi}}{A_{T}}=\frac{3.069}{225}=0.017$. (See Fig. 14).

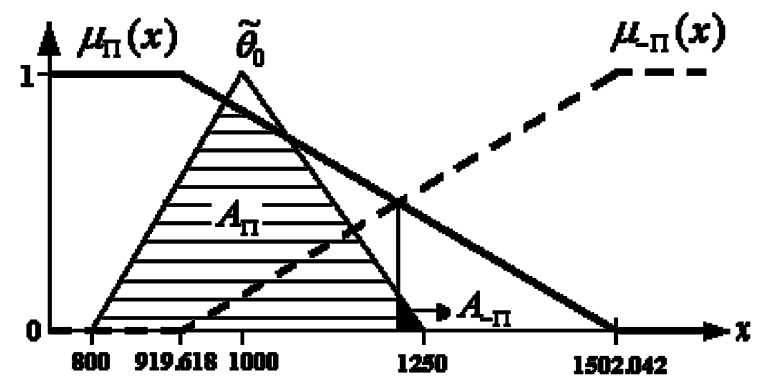

Fig. 14. $H_{0}$ is accepted (rejected) with $D A=0.962(D R=0.017)$.

\section{Conclusion}

The new approach proposed in this paper for testing statistical hypotheses in fuzzy environments, which is an extension of the Kahraman et al.'s work ${ }^{37}$, has certain merits as follows:

I) It is established upon the concept of fuzzy confidence interval. (Note that, in classical testing hypotheses, there is a close relationship between interval estimation and hypothesis testing ${ }^{39}$ ). 
II) By introducing the concepts of degree of acceptance $(D A)$ and degree of rejection $(D R)$, the procedure enables us to test fuzzy hypotheses in a rather natural way.

III) This work can be regarded as a step toward the computing with words in the field of statistical intelligent analysis.

Applications of the proposed method in fuzzy regression models ${ }^{41}$ and also in quality control ${ }^{42}$ may be some directions for future researches.

\section{Acknowledgments}

The authors would like to thank Professor R. Viertl for reading the manuscript and for his valuable suggestions and comments.

\section{References}

1. P.P. Bonissone, "Soft computing: the convergence of emerging reasoning technologies," Soft Computing, 1, 618 (1997).

2. L. Magdalena, "What is Soft Computing? Revisiting possible answers," International Journal of Computational Intelligence Systems, 3(2), 148-159 (2010).

3. R. Seising, "What is Soft Computing? Bridging gaps for 21 st Century science!," International Journal of Computational Intelligence Systems, 3(2), 160-175 (2010).

4. J. Liu, L. Martinez, H. Wang, R.M. Rodriguez, and V. Novozhilov, "Computing with words in risk assessment," International Journal of Computational Intelligence Systems, 3(4), 396-419 (2010).

5. L.A. Zadeh, "From computing with numbers to computing with words - from manipulation of measurements to manipulation of perceptions," IEEE Transactions on Circuits and Systems-1: Fundamental Theory and Applications, 45, 105-119 (1999).

6. M.R. Casals and M.A. Gil, "A note on the operativeness of Neyman-Pearson tests with fuzzy information," Fuzzy Sets and Systems, 30, 215-220 (1989).

7. M.R. Casals, M.A. Gil, and P. Gil, "The fuzzy decision problem: an approach to the problem of testing statistical hypotheses with fuzzy information," European Journal of Operation Research, 27, 371-382 (1986).

8. P. Grzegorzewski, "Testing statistical hypotheses with vague data," Fuzzy Sets and Systems, 112, 501-510 (2000).
9. H.Ch. Wu, "Statistical hypotheses testing for fuzzy data," Information Sciences, 175, 30-56 (2005).

10. R. Körner, "An asymptotic $\alpha$-test for the expectation of random fuzzy variables," Journal of Statistical Planning and Inference, 83, 331-346 (2000).

11. M. Montenegro, M.R. Casals, M.A. Lubiano, and M.A. Gil, "Two sample hypothesis tests of means of a fuzzy random variable," Information Sciences, 133, 89-100 (2001).

12. B.F. Arnold, "An approach to fuzzy hypothesis testing," Metrika, 44, 119-126 (1996).

13. B.F. Arnold, "Testing fuzzy hypotheses with crisp data," Fuzzy Sets and Systems, 94, 323-333 (1998).

14. S.M. Taheri and M. Arefi, "Testing fuzzy hypotheses based on fuzzy test statistic," Soft Computing, 13, 617625 (2009).

15. S.M. Taheri and J. Behboodian, "Neyman-Pearson Lemma for fuzzy hypothesis testing," Metrika, 49, 3-17 (1999).

16. S.M. Taheri and J. Behboodian, "A Bayesian approach to fuzzy hypotheses testing," Fuzzy Sets and Systems, 123, 39-48 (2001).

17. M. Arefi and S.M. Taheri, "Testing fuzzy hypotheses using fuzzy data based on fuzzy test statistic," Journal of Uncertain Systems, 5(1), 45-61 (2011).

18. P. Grzegorzewski, "Testing fuzzy hypotheses with vague data," In: Statistical Modeling, Analysis and Management of Fuzzy Data, Eds. C. Bertoluzza et al., Springer, Heidelberg, 213-225 (2002).

19. S.M. Taheri and J. Behboodian, "On Bayesian approach to fuzzy testing hypothesis with fuzzy data," Italian Journal of Pure and Applied Mathematics, 19, 139-154 (2006).

20. H. Torabi, J. Behboodian, and S.M. Taheri, "NeymanPearson Lemma for fuzzy hypotheses testing with vague data," Metrika, 64, 289-304 (2006).

21. R. Kruse and K.D. Meyer, "Statistics with Vague Data," Reidel Publ, Dordrecht, Netherlands, (1987).

22. H. Torabi, "A generalized version of Neyman-Pearson Lemma for testing fuzzy hypotheses based on r-level sets," Communications in Statistics: Theory and Methods, 41(24), 4379-4390 (2012).

23. P. Filzmoser and R. Viertl, "Testing hypotheses with fuzzy data: the fuzzy P-value," Metrika, 59, 21-29 (2004).

24. A. Parchami, S.M. Taheri, and M. Mashinchi, "Fuzzy pvalue in testing fuzzy hypotheses with crisp data," Statistical Papers, 51, 209-226 (2010).

25. A. Parchami, S.M. Taheri, and M. Mashinchi, "Testing fuzzy hypotheses based on vague observations: a p-value approach," Statistical Papers, 53(2), 469-484 (2012).

26. M. Arefi and S.M. Taheri, "A fuzzy-based approach to testing statistical hypotheses," International Journal of Intelligent Technologies and Applied Statistics, 4(1), 109-132 (2011). 
27. J.J. Buckley, "Fuzzy statistics: hypothesis testing," Soft Computing, 9, 512-518 (2004).

28. J. Chachi, S. Taheri, and R. Viertl, "Testing statistical hypotheses based on fuzzy confidence intervals," Austrian Journal of Statistics, 41(4), 267-286 (2012).

29. P. Grzegorzewski, "K-sample median test for vague data," International Journal of Intelligent Systems, 24, 529-539 (2009).

30. P. Grzegorzewski, and O. Hryniewicz, "Testing statisti- 3 cal hypotheses in fuzzy environment," Mathware \& Soft Computing, 4, 203-217 (1997).

31. O. Hryniewicz, "Goodman-Kruskal $\gamma$ measure of depen- 39. dence for fuzzy ordered categorical data," Computational Statistics and Data Analysis, 51, 323-334 (2006).

32. S.M. Taheri and G. Hesamian, "A generalization of the Wilcoxon signed-rank test and its applications," Statistical Papers, (to appear), Doi: 10.1007/s00362-012-04434, (2012).

33. S.M. Taheri and G. Hesamian, "Goodman-Kruskal measure of association for fuzzy-categorized variables," $K y$ bernetika, 47(1), 110-122 (2011).

34. S.M. Taheri, "Trends in fuzzy statistics," Austrian Journal of Statistics, 32, 239-257 (2003).

35. R. Viertl, "Statistical Methods for Fuzzy Data”, J. Wiley,
Chichester (2011).

36. R. Viertl, "Fuzzy models for precision measurements," Mathematics and Computers in Simulation, 79, 874-878 (2008).

37. C. Kahraman, C.E. Bozdag, D. Ruan, and A.F. Ozok, "Fuzzy sets approaches to statistical parametric and nonparametric tests," International Journal of Intelligent Systems, 19, 1-19 (2004).

8. G.J. Klir and B. Yuan, "Fuzzy Sets and Fuzzy Logic Theory and Applications," Prentic-Hall, Englewood Cliffs, NJ, (1995).

9. G. Casella and R.L. Berger, "Statistical Inference”, (Second Edition), Pacific Grove, CA 93950 USA, Duxbury, (2002).

40. J. Chachi and S.M. Taheri, "Fuzzy confidence intervals for mean of Gaussian fuzzy random variables," Expert Systems with Applications, 38(5), 5240-5244 (2011).

41. S. Sentürk, "Fuzzy regression control chart based on $\alpha$ cut approximation," International Journal of Computational Intelligence Systems, 3(1), 123-140 (2010).

42. M.H.F. Zarandi, A. Alaeddini, and I.B. Turksen, "A hybrid fuzzy adaptive sampling Run rules for Shewhart control charts," Information Sciences, 178(4), 1152-1170 (2008). 